American Journal of Applied Sciences 7 (2): 201-207, 2010

ISSN 1546-9239

(C) 2010 Science Publications

\title{
Effects of N-Acetylcysteine on Auditory Brainstem Response Threshold Shift in Rabbits Exposed to Noise and Carbon Monoxide
}

\author{
${ }^{1}$ Seyedbagher Mortazavi, ${ }^{1}$ Masoud Motalebi Kashani, ${ }^{1}$ Ali Khavanin, \\ ${ }^{2}$ Abdolamir Alameh, ${ }^{3}$ Ramezan Mirzaee and ${ }^{4}$ Mehdi Akbari \\ ${ }^{1}$ Department of Occupational and Environmental Health, School of Medicine, \\ Tarbiat Modares University, Tehran, Iran \\ ${ }^{2}$ Department of Biochemistry, School of Medicine, \\ Tarbiat Modares University, Tehran, Iran \\ ${ }^{3}$ Department of Occupational Health, School of Health, \\ Zahedan University of Medical Sciences, Zahedan, Iran \\ ${ }^{4}$ Department of Audiology, School of Rehabilitation Sciences, \\ Iran University of Medical Sciences, Tehran, Iran
}

\begin{abstract}
Problem statement: Noise Induced Hearing Loss (NIHL) is one of the most important occupational disease in worldwide. NIHL has been found to be potentiated by simultaneous Carbon monoxide (CO) exposure. Exposure to noise plus $\mathrm{CO}$ is common in occupational and environmental settings. Free radicals have been implicated in cochlear damage resulting from exposure to noise and due to CO hypoxia This study examined whether N-Acetylcysteine (NAC) administration cause attenuation of Auditory Brainstem Response (ABR) threshold shifts resulting from noise exposure and noise plus $\mathrm{CO}$ exposure. Approach: Forty-two rabbits were divided into seven groups including control, noise + saline, noise $+\mathrm{CO}+$ saline, noise $+\mathrm{NAC}$, noise $+\mathrm{CO}+\mathrm{NAC}, \mathrm{CO}+\mathrm{NAC}$ and NAC alone. ABR was assessed before exposure, 1 hour and 14 days post exposure. Results: The administration of $325 \mathrm{mg} \mathrm{kg}{ }^{-1}$ of NAC prior, following and post exposure to noise or noise plus CO recovered permanent $\mathrm{ABR}$ threshold shift at 1 and $2 \mathrm{kHz}$ almost to the baseline and provided significant attenuation in permanent $\mathrm{ABR}$ threshold shift at 4 and $8 \mathrm{kHz}$ in subjects which were exposed to noise but it did not block the potentiating of threshold elevation by $\mathrm{CO}$ exposure (extra threshold loss by combined exposure) at 4 and $8 \mathrm{kHz}$. Conclusion: NAC provides protective effect against hearing loss resulting from noise exposure and simultaneous exposure to noise plus $\mathrm{CO}$.
\end{abstract}

Key words: Noise induced hearing loss, antioxidant agent, NIHL potentiation, auditory brainstem response, NAC

\section{INTRODUCTION}

Excessive noise can cause many health problems in the workplace but Noise-Induced Hearing Loss (NIHL) is the most important effect of noise on the auditory system in occupational settings. NIHL is a widespread disease in the world with $16 \%$ of the disabling hearing loss in adults being attributed to occupational noise (Nelson et al., 2005). NIHL has been found to be potentiated by simultaneous exposure to noise and Carbon monoxide (CO), while $\mathrm{CO}$ exposure alone may not induce permanent hearing loss (Chen and Fechter, 1999; Rao and Fechter, 2000a). CO is the most abundant pollutant in air and exposure to noise plus $\mathrm{CO}$ is common in many work environments. The mechanisms of NIHL can be classified into two major categories: direct mechanical trauma and metabolic damage to the organ of corti. The generation of Reactive Oxygen Species (ROS) is considered to be part of the second mechanism (Prell et al., 2007; Heinrich and Feltens, 2006; Fechter, 2005).

Early reports of noise-induced free radical formation in the cochlea led to the hypothesis that NIHL can be attenuated by exogenous antioxidants as well as endogenous ROS scavengers. Many compounds have been used for the prevention and treatment of NIHL (Kopke et al., 2005; Lynch and Kil, 2005). The mechanism underlying NIHL potentiation by $\mathrm{CO}$ is

Corresponding Author: Seyedbagher Mortazavi, Department of Occupational and Environmental Health, School of Medicine, Tarbiat Modares University, Tehran, Iran 
unclear but combined exposure to noise and $\mathrm{CO}$ is believed to increase free radical levels in the cochlea (Fechter et al., 2002; Rao and Fechter, 2000b). In many cases, engineering and administrative measures for reduction of noise and $\mathrm{CO}$ levels is very difficult; however, hearing protection devices is seldom used by workers because of inherent limitation. Hence, a pharmacological preventative measure for NIHL can be an important element of hearing conservation programs in the workplace. Many studies have demonstrated that $\mathrm{N}$-Acetylcysteine (NAC) as an antioxidant and as a substrate for glutathione synthesis can effectively reduce NIHL in animal models (Kopke et al., 2000; 2004; 2005).

The present study has been designed to test the hypothesis that injury resulting from simultaneous exposure to noise plus $\mathrm{CO}$ is reduced by NAC in a rabbit model.

\section{MATERIALS AND METHODS}

Animals: Healthy male adult white New Zealand rabbits (2200-2500 g body weight) were acquired from the Pasture Institute of Iran. Animals were examined for possible otitis media prior to auditory assessments. Rabbits were maintained on a $12 \mathrm{~h}$ light $/ 12 \mathrm{~h}$ dark cycle at $23-27^{\circ} \mathrm{C}$ and they had free access to food (Purina laboratory chow) and water. All procedures regarding the use and handling of animals were carried out in accordance with the principles of Helsinki and in accordance with animal use protocols approved by the appropriate institutional animal care and use committees.

Experimental protocol: Forty-two rabbits were divided into seven groups ( $\mathrm{n}=6$, each group) according to type of experiment as follows: Group 1, control (no exposure to noise or $\mathrm{CO}$ and no injection); Group 2, exposed to noise alone and received saline intrapretoneal (i.p.) injection; Group 3, exposed to noise plus $\mathrm{CO}$ and received saline i.p. injection; Group 4, exposed to noise alone and received NAC $325 \mathrm{mg} \mathrm{kg}^{-1}$ body weight i.p. injection; Group 5, exposed to noise plus $\mathrm{CO}$ and received NAC $325 \mathrm{mg} \mathrm{kg}^{-1}$ body weight i.p. injection; Group 6, not exposed to noise or $\mathrm{CO}$ and received NAC $325 \mathrm{mg} \mathrm{kg}^{-1}$ body weight i.p. injection; Group 7, exposed to $\mathrm{CO}$ alone and received saline i.p. injection. The total duration of experimental procedures was 22 days. Noise and $\mathrm{CO}$ exposure was conducted for 5 consecutive days on day 4 through day 8 . NAC or saline was injected once a day from day 1 through day 11 (3 days before the exposure, 5 days during the exposure and 3 days after exposure). Auditory Brain- stem Response (ABR) threshold at 1, 2, 4 and $8 \mathrm{kHz}$ were measured at three time points: day 1 (prior to experiment), day 8 (immediately at the end of exposure) and day 22 (14 days after exposure).

The exposure chamber was designed as a reverberant field which could accommodate six rabbits at a time. The chamber was equipped with stereo speakers for delivering noise. Air exchange rate in this chamber was adjusted to 12 air changes per hour using an exhaust fan. The ventilation system generated noise below $40 \mathrm{~dB}$ which was masked by the noise generated for exposure experiments. Experimental Group 2-5 were exposed to octave band noise centered at $4 \mathrm{kHz}, 100 \mathrm{~dB}$ SPL, $8 \mathrm{~h}$ day $^{-1}$ for 5 days consecutively. This octave band noise was generated and controlled with a custom software program (signal software) and then amplified and delivered with a computer system using Cool Edit software to two load speakers. Sound pressure level in the chamber was measured using a precision sound level meter (Cel$460, \mathrm{UK})$ at the level of animal head $(15 \mathrm{~cm}$ above the chamber floor). Noise exposure levels in the chamber were monitored every $30 \mathrm{~min}$. The variability of the noise within the animals' roaming area was less than $2 \mathrm{~dB}$.

Animals exposed to noise plus $\mathrm{CO}$ or $\mathrm{CO}$ alone based on experimental design. The $\mathrm{CO}$ concentration in the exposure chamber was $700 \mathrm{ppm}$ with an actual level achieved of $700 \pm 25 \mathrm{ppm}$ (SD). Previous studies have shown that carboxyhemoglobin levels approach steady state within $30 \mathrm{~min}$ of exposure onset and have stabilized after $90 \mathrm{~min}$ of exposure (Chen and Fechter, 1999). Therefore, CO exposure began $90 \mathrm{~min}$ prior to the onset of noise to assure equilibration of carboxyhemoglobin. The total daily exposure time to noise was $8 \mathrm{~h}$ and the daily exposure time to $\mathrm{CO}$ was $9.5 \mathrm{~h}(8+1.5 \mathrm{~h})$. Experimental Group 3, 5 and 7 were exposed to $\mathrm{CO}$ at $700 \mathrm{ppm}$ concentration, $9.5 \mathrm{~h} \mathrm{day}^{-1}$ for 5 days consecutively. $\mathrm{CO}$ gas was delivered into the chamber using a micro valve (Air Flow) and the $\mathrm{CO}$ level in the chamber was measured according to a standard procedure described in NIOSH: Method No 6604 by carbon monoxide monitor STICK (testo 317-3) (National Institute of Occupational safety and Health, 1999). The concentration of CO was monitored continuously. Experimental Group 4-6 received NAC325 mg kg${ }^{-1}$ body weight once a day for 11 days by i.p. injection. During the exposure days, NAC was given $1 \mathrm{~h}$ prior to noise or noise plus CO exposure. Group 1-3 and 7 received saline with a similar volume and schedule. Protective effect of NAC against CO alone was not studied since $\mathrm{CO}$ alone does not cause any permanent auditory impairment (Chen and Fechter, 1999). 
Auditory brainstem response testing: Auditory function was evaluated by Auditory Brainstem Response (ABR) threshold test. Before ABR test, animals were anesthetized with Xylazine $\left(10 \mathrm{mg} \mathrm{kg}^{-1}\right.$ body weight) and ketamin (40 $\mathrm{mg} \mathrm{kg}^{-1}$ body weight) mixture by i.m. injection. The external ear canals and tympanic membranes were inspected to ensure the ear canal was free of wax. The body temperature of rabbits was maintained at $37 \pm 1^{\circ} \mathrm{C}$ using a heating blanket. An active needle electrode was inserted subcutaneously below the test ear, a reference electrode at the vertex and a ground electrode below the other ear. The test stimuli were $1,2,4$ and $8 \mathrm{kHz}$ tone bursts $(1 \mathrm{~ms}$ Blackman rise/fall, $15 \mathrm{~ms}$ duration, alternating polarity) generated using Interacoustics EP25. Stimuli were routed through an insert phone fitted in the rabbit's ear canal. Near the threshold, the sound intensity was decreased in $5 \mathrm{~dB}$ steps and responses for 1024 tone presentations at $20 / \mathrm{s}$ were averaged at each intensity level. ABR threshold was defined as the lowest stimulus intensity that produces a reproducible ABR waveform peak 3 or 4 . The average threshold of the right and left ear at a specific frequency was considered as the ABR threshold for a specific time point. All experimental groups were tested prior to experiment on day 1 to establish the baseline ABR threshold, day 11 (immediately at the end of exposure) and day 22 (14 days after exposure). Temporary Threshold Shift (TTS) was calculated at each frequency as the difference between baseline ABR threshold and ABR threshold on day 11 (0 day post exposure). The difference between baseline ABR threshold and ABR threshold on day 22 at each frequency was considered as the Permanent Threshold Shift (PTS).

Non-protein GSH measurement: Blood Glutathione (GSH) level was measured to evaluate the effects of NAC on glutathione synthesis in rabbit and compare blood GSH levels from control and treated rabbits. Total blood GSH was measure using Ellman's reagent (Mirzaee et al., 2007). The whole blood (0.2 mL) was taken in a $10 \mathrm{~mL}$ test tube and was mixed by adding 1.8 $\mathrm{mL}$ of distilled water. Then $3.0 \mathrm{~mL}$ of the precipitation solution (1.67 $\mathrm{g}$ of glacial metaphosphoric acid, $0.2 \mathrm{~g}$ of disodium-EDTA and $30 \mathrm{~g}$ of $\mathrm{NaCl}$ in $100 \mathrm{~mL}$ water) was added. The mixture was allowed to stand for $5 \mathrm{~min}$ at room temperature and then filtered to remove proteins. Exactly $0.8 \mathrm{~mL}$ of the filtrate was mixed with $1.0 \mathrm{~mL}$ of DTNB (40 mg DTNB in $100 \mathrm{~mL}$ of sodium citrate solution). The intensity of the color was recorded at $412 \mathrm{~nm}$ using Shimadzu UV-3100 double beam spectrophotometer. The concentration of GSH in samples was calculated from a calibration curve which was prepared from freshly prepared standard solution of GSH.

This assay was performed at two time points: Day 4 (3 days after receiving NAC or saline and prior to noise or $\mathrm{CO}$ exposure) and day 8 ( 8 days after receiving NAC or saline and 5 days post exposure to noise or noise plus $\mathrm{CO}$ ).

Statistical analysis: The Kolmogorov-Smirnov test was used to assess the normality of data in each group. The ABR threshold shift at each frequency was compared among groups using one-way Analysis of Variance (ANOVA) and the Tukey test was applied as a post hoc test for multiple comparisons between groups. Likewise, ANOVA was used for comparisons of ABR threshold shift between frequencies at each group and comparison of blood GSH level among groups. All statistical tests were performed using SPSS 15 for windows and $\mathrm{p}<0.05$ was considered significant.

\section{RESULTS}

Blood Glutathione (GSH): The results indicate the blood GSH level in subjects which received NAC (Group 4-6) was significantly more than other groups on day 4 (prior to exposure) and day 8 (5 days after exposure). Blood GSH level on day 8 was found to be reduced significantly in Group 2, 3 and 7 as compared to Group 1 (control) (Fig. 1).

ABR measurement: Baseline ABR thresholds at each frequency were similar in all groups. The baseline ABR threshold at 8 and $4 \mathrm{kHz}$ were significantly smaller than 2 and $1 \mathrm{kHz}$ in all groups. ABR threshold shift on day 8 ( $1 \mathrm{~h}$ after exposure) and day 22 (14 days after exposure) at each frequency in experimental groups are represented in Fig. 2.

The ABR threshold shift of the control group was approximately 0 on day 8 and day 22. Statistical comparisons showed that the ABR threshold shifts of Group 6 (unexposed and received NAC) and Group 7 (CO exposure and received saline) at each frequency were not significantly different from Group 1 (control) at all time points. This indicates that $\mathrm{CO}$ exposure alone and receiving NAC alone could not alter ABR thresholds. The ABR threshold of Group 2 (noise + saline) had significant elevation compared to the control group at all frequencies on day 8 and day 22 but was significantly smaller than the ABR threshold of Group 3 (noise $+\mathrm{CO}+$ saline) $(\mathrm{p}<0.01)$. 


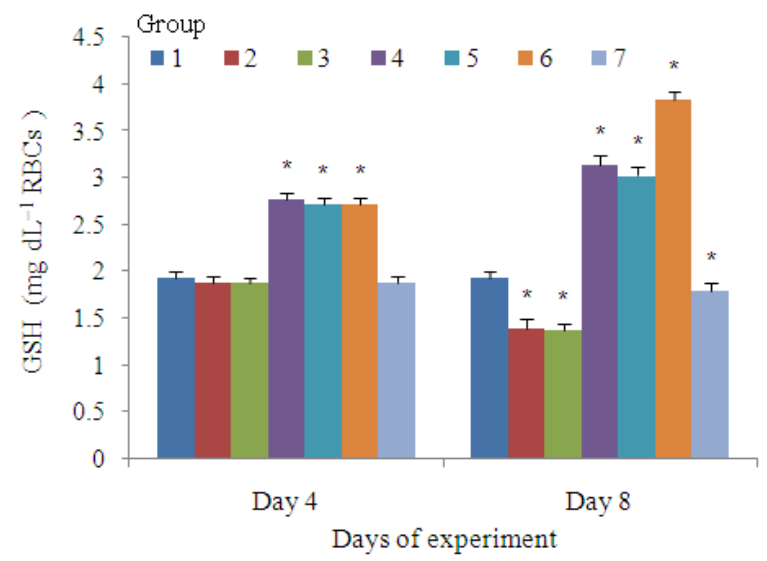

Fig. 1: Blood Glutathione (GSH) levels on day 4 (prior to exposure) and day 8 (5 days after exposure) in each group. Bars show means, error bars show \pm 1.0 SD. $\quad *$ : $p<0.05$, significantly different from Group 1. Groups: (1) control (no exposure to noise or $\mathrm{CO}$, and no i.p. injection); (2) noise exposure + saline i.p. injection; (3) noise plus $\mathrm{CO}$ exposure + saline i.p. injection; (4) noise exposure + NAC $325 \mathrm{mg} \mathrm{kg}^{-1}$ i.p. injection; (5) noise plus $\mathrm{CO}$ exposure + NAC $325 \mathrm{mg} \mathrm{kg}^{-1}$ i.p. injection; (6) no exposure to noise or $\mathrm{CO}+\mathrm{NAC} 325 \mathrm{mg} \mathrm{kg}^{-1}$ i.p. injection; (7) $\mathrm{CO}$ exposure + saline i.p. injection

The saline + noise group (Group 2) had a $44.8 \mathrm{~dB}$ threshold loss on day 8 and $20.1 \mathrm{~dB}$ threshold loss on day 22 averaged across frequencies of $1-8 \mathrm{kHz}$ while animals receiving saline + noise $+\mathrm{CO}$ (Group 3 ) had a 58.5 and $28.6 \mathrm{~dB}$ average threshold loss on day 8 and day 22 respectively. The ABR threshold shifts of Group 3 at 4 and $8 \mathrm{kHz}$ were significantly more prominent than at 1 and $2 \mathrm{kHz}$ at all time points tested $(\mathrm{p}<0.01)$.The noise-induced ABR threshold shifts at all frequencies were found to be reduced significantly in animals which were exposed to noise and received NAC (Group 4) compared to subjects which were exposed to noise and received saline (Group 2) on day 8 and day $22(p<0.01)$. This reduction at 1 and $2 \mathrm{kHz}$ was significantly more than at 4 and $8 \mathrm{kHz}$ $(p<0.01)$. The average threshold elevation across frequencies $1-8 \mathrm{kHz}$ of Group 4 was $27.7 \mathrm{~dB}$ on day 8 and $5.6 \mathrm{~dB}$ on day 22 .

The ABR thresholds of animals in Group 4 at all frequencies were significantly greater than that of control group, $1 \mathrm{~h}$ after exposure while 14 days after exposure the thresholds at 1 and $2 \mathrm{kHz}$ recovered almost to the baseline.

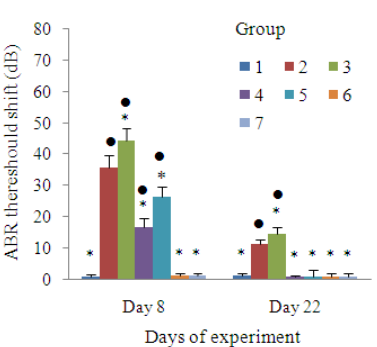

(A)

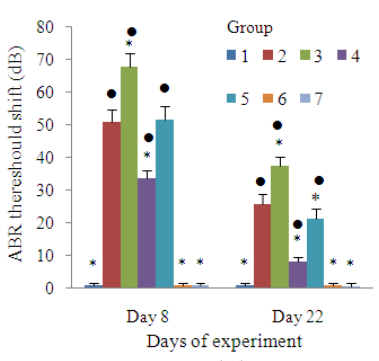

(C)

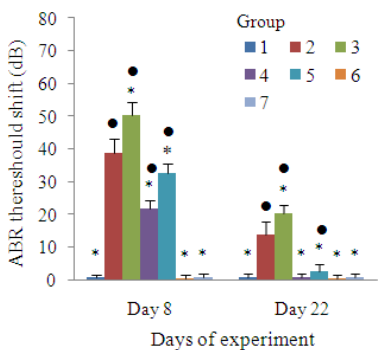

(B)

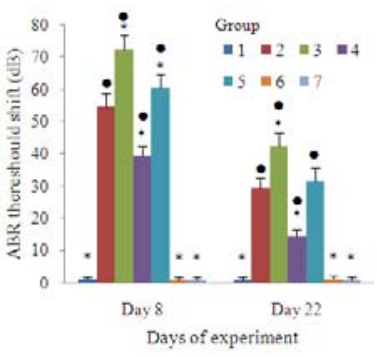

(D)
Fig. 2: ABR threshold shifts on day 8 ( $1 \mathrm{~h}$ post exposure to noise or noise plus $\mathrm{CO}$ ) and day 22 (14 days post exposure to noise or noise plus $\mathrm{CO}$ ) in each group at $1 \mathrm{KHz}(\mathrm{A}), 2 \mathrm{KHz}(\mathrm{B})$, $4 \mathrm{kHz}(\mathrm{C})$ and $8 \mathrm{KHz}(\mathrm{D})$. Bars show means, error bars show \pm 1.0 SD. *: $\mathrm{p}<0.05$, significantly different from group $2 .^{\bullet}: \mathrm{p}<0.05$, significantly different from Group 1. Groups: (1) control (no exposure to noise or $\mathrm{CO}$, and no i.p. injection); (2) noise exposure + saline i.p. injection; (3) noise plus $\mathrm{CO}$ exposure + saline i.p. injection; (4) noise exposure $+\mathrm{NAC}$ $325 \mathrm{mg} \mathrm{kg}^{-1}$ i.p injection; (5) noise plus $\mathrm{CO}$ exposure + NAC $325 \mathrm{mg} \mathrm{kg}^{-1}$ i.p. injection; (6) no exposure to noise or $\mathrm{CO}+\mathrm{NAC} 325 \mathrm{mg} \mathrm{kg}^{-1}$ i.p. injection; (7) $\mathrm{CO}$ exposure + saline i.p. injection

The ABR threshold of animals exposed to noise plus $\mathrm{CO}$ and treated with NAC (Group 5) at all frequencies was significantly smaller than that of animals which were exposed to noise plus $\mathrm{CO}$ and which received saline (Group 3) on day 8 and day 22. This indicates that treatment with NAC could reduce the temporary and permanent ABR threshold shift at all frequencies in subjects exposed to noise and $\mathrm{CO}$ simultaneously.

The ABR threshold loss of Group 5 was $42.5 \mathrm{~dB}$ on day 8 and $13.8 \mathrm{~dB}$ on day 22 averaged across frequencies 1-8 $\mathrm{kHz}$. These values were significantly different from the control group $(\mathrm{p}<0.01)$. Comparison among experimental groups in Fig. 2 suggest that NAC 
could attenuate ABR threshold shifts in animals exposed to noise plus $\mathrm{CO}$, but it did not block the potentiating of threshold elevation by $\mathrm{CO}$ exposure (extra threshold loss by combined exposure) at $8 \mathrm{kHz}$ on day 8.

\section{DISCUSSION}

The results showed that, when NAC was administered 3 days prior, following and 3 days post exposure to noise (or noise plus $\mathrm{CO}$ ) by i.p. injection (325 mg kg $\mathrm{m}^{-1}$ body weight), adequate protection was provided against noise induced hearing loss and appropriate protection was provided against combined exposure-induced hearing loss in rabbits.

Rabbits were used as the animal model in this investigation because the hearing frequency range is approximately $360-42000 \mathrm{~Hz}$, which covers the frequency range of humans. Octave band noise centered at $4 \mathrm{kHz}, 100 \mathrm{~dB}$ SPL, $8 \mathrm{~h} \mathrm{day}{ }^{-1}$ for 5 days consecutively was chosen to imitate continuous noise in the workplace. Likewise, maximum potentiation of NIHL by CO was reported at $100 \mathrm{~dB}$ SPL (Fechter et al., 2000).

However significant potentiation of NIHL by CO was reported with $500 \mathrm{ppm}$ and higher concentrations of $\mathrm{CO}$ in a previous study (Fechter et al., 2000). Preliminary experiments demonstrate at least $700 \mathrm{ppm}$ of $\mathrm{CO}$ exposure is required to provide more $\mathrm{ABR}$ threshold elevation than the noise alone. Rabbits exposed to simultaneous noise and $\mathrm{CO}$ had further elevation in temporary and permanent threshold shift at all frequencies as compared with those exposed to noise. This phenomenon was reported in several previous studies (Chen and Fechter, 1999; Fechter et al., 2002).

Administration of NAC was begun 3 days before noise (or noise plus $\mathrm{CO}$ ) exposure for providing a stable and higher concentration of glutathione in the cochlea at beginning of the exposure. Biochemical assay showed that the blood GSH level at groups that received NAC were increased as compared with other groups at prior to exposure. Previous studies have indicated that GSH is an important factor in the modification of inner ear trauma following noise exposure (Ohinata et al., 2000; Yamasoba et al., 1998). However, the depletion of blood Glutathione (GSH) in rabbits exposed to noise has been previously reported (Mirzaee et al., 2007). NAC was given 3 days post exposure because there is evidence for delayed free radical formation, peaking 7-10 days following noise exposure and the finding that free radical scavengers administered as long as 3 days post-noise attenuate free radical formation and reduce NIHL (Prell et al., 2007).
NAC was administrated $325 \mathrm{mg} \mathrm{kg}^{-1}$ body weight by i.p. injection because many studies indicated that a dose of $325 \mathrm{mg} \mathrm{kg}^{-1}$ was most effective for attenuation of NIHL (Kopke et al., 2007; Duan et al., 2004).

Results from this study indicate that NAC attenuated temporary $\mathrm{ABR}$ threshold shift more significantly at 1 and $2 \mathrm{kHz}$ than at 4 and $8 \mathrm{kHz}$ in subjects exposed to noise (or noise plus $\mathrm{CO}$ ) while noise (or noise $+\mathrm{CO}$ ) could cause more temporary ABR threshold shift at 4 and $8 \mathrm{kHz}$ than at 1 and $2 \mathrm{kHz}$. This finding is consistent with previous research showing that antioxidant agents were more effective at the frequencies away from the frequencies with greater threshold shift (Ohinata et al., 2000 ; Hou et al., 2003; Yamasoba et al., 1999) This may be because other factors, in addition to ROS formation.

The current work showed NAC recovered permanent $\mathrm{ABR}$ threshold shift at 1 and $2 \mathrm{kHz}$ almost to the baseline and provided significant attenuation in permanent $\mathrm{ABR}$ threshold shift at 4 and $8 \mathrm{kHz}$ in subjects exposed to noise. NAC as a GSH prodrug and antioxidant can ameliorate the cochlear damage through a variety of mechanisms, such as providing a substrate for cochlear GSH synthesis, free radical scavenging and inhibition of CDP pathway activation and necrosis.

The results showed that NAC can significantly attenuate the permanent $\mathrm{ABR}$ threshold shift in subjects exposed to simultaneous noise and $\mathrm{CO}$.

We know that two major mechanisms are involved in NIHL including mechanical damage and metabolic alternations, but the mechanism of the potentiation of NIHL by $\mathrm{CO}$ is still unclear. $\mathrm{CO}$ exposure causes reduction of oxygen supply and makes recovery of outer hair cell function impossible, while subjects exposed to noise alone are capable of recovery from a temporary threshold shift (Chen and Fechter, 1999). Many studies indicated that an excitotoxic mechanism may be involved in $\mathrm{CO}$ ototoxicity such as $\mathrm{CO}$ inducing excessive glutamate release (Fechter et al., 1997). Excessive glutamate release has been identified as a basic mechanism of neurotoxicity through the process of excitotoxicity (Chen et al., 2001). Free radical generation seems also involved in $\mathrm{CO}$ ototoxicity. Production of hydroxyl radicals has been demonstrated directly in the hippocampus due to $\mathrm{CO}$ hypoxia (Piantadosi et al., 1997).

NAC-induced GSH synthesis may also be able to ameliorate the effects of glutamate excitotoxicity as well as protect cochlear mitochondria from injury. NAC has been shown to be an effective scavenger of hydroxyl radical (Kopke et al., 2007).

NAC, a thiol-containing amino acid derivative, is used in the United States as a nutritional supplement 
and also a drug which has passed the stringent safety requirements for Food and Drug Administration (FDA) approval for prescription use only.

Thus, NAC may be an excellent candidate for the prevention of NIHL in humans. It must be mentioned that these findings are limited due to the one time point and one dosage level administration of NAC. Further studies of its action are needed.

\section{CONCLUSION}

NAC (325 mg kg ${ }^{-1}$, i.p. injection) can attenuate temporary and permanent noise induced ABR threshold shifts and provides appropriate protection against $A B R$ threshold shift resulting from exposure to noise plus $\mathrm{CO}$ in rabbits.

\section{ACKNOWLEDGEMENT}

This research was supported by Tarbiat Modares University.

\section{REFERENCES}

Chen, G.D. and L.D. Fechter, 1999. Potentiation of octave-band noise induced auditory impairment by carbon monoxide. Hear. Res., 132: 149-59. DOI: 10.1016/s0378-5955(99)00044-1

Chen, G.D., Y. Kong, K. Reinhard and L.D. Fechter, 2001. NMDA receptor blockage protects against permanent noise-induced hearing loss but not its potentiation by carbon monoxide. Hear. Res., 154: $\quad 108-115 . \quad$ DOI: $10.1016 / \mathrm{s} 0378-$ 5955(01)00226-X

Duan, M., J. Qiu, G. Laurell, A. Olofsson and S.A. Counter et al., 2004. Dose and timedependent protection of the antioxidant N-Lacetylcysteine against impulse noise trauma. Hear. Res., 192: 1-9. DOI: 10.1016/j.heares.2004.02.005

Fechter, L.D., 2005. Oxidative stress: A potential basis for potentiation of noise-induced hearing loss. Environ. Toxicol. Pharmacol., 19: 543-546. DOI: 10.1016/j.etap.2004.12.017

Fechter, L.D., G.D. Chen and D. Rao, 2002. Chemical asphyxiants and noise. Noise Health, 4: 49-61. PMID: 12678928

Fechter, L.D., G.D. Chen, D. Rao and J. Larabee, 2000. Predicting exposure conditions that facilitate the potentiation of noise-induced hearing loss by carbon monoxide. Toxicol. Sci., 58: 315-323. http://toxsci.oxfordjournals.org/cgi/content/abstract /58/2/315
Heinrich, U. and R. Feltens, 2006. Mechanisms underlying noise-induced hearing loss. Drug discovery today: Dis. Mechanisms, 3: 131-135. DOI: 10.1016/j.ddmec.2006.02.003

Hou, F., S. Wang, S. Zhai, Y. Hu, W. Yang and L. He, 2003. Effects of $\alpha$-tocopherol on noise-induced hearing loss in guinea pigs. Hear. Res., 179: 1-8. DOI: 10.1016/s0378-5955 (03)00065-0

Kopke, R., E. Bielefeld, Y. liu, J. Zheng and R. Jackson, 2005. Prevention of impulse noise-induced hearing loss with antioxidants. Acta otolaryngol, 125: 235-243. http://www.informa.com

Kopke, R.D., E. Blelefeld, Y. liu, J. Zheng and R. Jackson, 2004. NAC and Acetyle-L-carnitine (ALCAR) show different effects in protecting the cochlea from noise in chinchilla. Assoc. Res. Otolaryngol. Abs., 27: 231. http://www.aro.org/abstracts

Kopke, R.D., P.A. Weisskopf, J.L. Boon, R.L. Jackson and D.C. Wester et al., 2000. Reduction of noiseinduced hearing loss using NAC and salicylate in the chinchilla. Hear. Res., 149: 138-146. DOI: 10.1016/s0378-5955(00)00176-3

Kopke, R.D., R.L. Jackson, J.K.M. Coleman, J. Liu and E.C. Bielefelde et al., 2007. NAC for noise: From the bench top to the clinic. Hear. Res., 226: 114125. DOI: 10.1016/j.heares.2006.10.008

Fechter, L.D., Y. Liu and T.A. Pearce, 1997. Cochlear protection from carbon monoxide exposure by free radical blockers in the guinea pig. Toxicol. Applied Pharmacol., 142: 47-55. DOI: 10.1006/taap.1996.8027

Lynch, E.D. and J. Kil, 2005. Compounds for the prevention and treatment of noise-induced hearing loss. Drug Discov. Today, 10: 1291-1298. DOI: 10.1016/s1359-6446(05)03561-0

Mirzaee, R., A. Allameh, S.B. Mortazavi, A. Khavanin and A. Kazemnejad et al., 2007. Assessment of outer hair cell function and blood antioxidant status of rabbits exposed to noise and metal welding fumes. Auris Nasus Larinx, 34: 147-154. DOI: 10.1016/j.anl.2006.07.003

National Institute for Occupational safety and Health (NIOSH), 1999. NIOSH, Manual of Analytical Methods. Environmental Monitoring Systems. http://www.cdc.gov/niosh/docs/2003154/pdfs/6604.pdf

Nelson, D.I., R.Y. Nelson, M. Concha-Barrientons and M. Fingerhut, 2005. The global burden of occupational noise-induced hearing loss. Am. J. India Med., 48: 446-458. DOI: 10.1002/ajim.20223

Ohinata, Y., T. Yamasoba, J. Schacht and J.M. Miller, 2000. Glutathione limits noise-induced hearing loss. Hear. Res., 146: 28-34. DOI: 10.1016/s03785955(00)00096-4 
Piantadosi, C.A., J. Zhang and I.T. Demchenko, 1997. Production of hydroxyl radical in the hippocampus after CO hypoxia or hypoxic hypoxia in the rat. Free. Radical. Biol. Med., 22: 725-732. DOI: 10.1016/s0891-5849(96)00423-6

Prell, C.G., D. Yamashita, S.B. Minami, T. Yamasoba and J.M. Miller, 2007. Mechanisms of noiseinduced hearing loss indicate multiple methods of prevention. Hear. Res., 226: 22-43. DOI: 10.1016/j.heares.2006.10.006

Rao, D.B. and L.D. Fechter, 2000a. Increasing noise severity limits poetntiation of noise induced hearing loss by carbon monoxide. Hear. Res., 150: 206-214. DOI: 10.1016/s0378$5955(00) 00202-1$
Rao, D. and L.D. Fechter, 2000b. Protective effects of phenyl-N-tert butylnitrone on the potentiation of noise-induced hearing loss by carbon monoxide. Toxicol. Applied Pharmacol., 167: 125-131. DOI: 10.1006/taap.2000.8995

Yamasoba, T., A.L. Nuttall, C. Harris, Y. Raphael and J.M. Miller, 1998. Role of glutathione in protection against noise-induced hearing loss. Brain Res., 784: 82-90. DOI: 10.1016/s0006-8993(97)01156-6

Yamasoba, T., J. Schacht, F. Shoji and J.M. Miller, 1999. Attenuation of cochlear damage from noise trauma by an iron chelator, a free radical scavenger and glial cell line-derived neurotrophic factor in vivo. Brain. Res., 815: 317-325. DOI: 10.1016/s0006-8993 (98)01100-7 